\title{
Improvement of the Handover Performance and Channel Allocation Scheme using Fuzzy Logic, Artificial Neural Network and Neuro-Fuzzy System to Reduce Call Drop in Cellular Network
}

\author{
Md. Ariful Islam ${ }^{1, *}$, Md. Rakib Hasan ${ }^{2}$ and Amena Begum ${ }^{2}$ \\ ${ }^{1}$ Department of Robotics \& Mechatronics Engineering, University of Dhaka, Dhaka, Bangladesh \\ ${ }^{2}$ Department of Information \& Communication Technology, Comilla University, Cumilla, Bangladesh
}

Received: November 27, 2020, Revised: December 16, 2020, Accepted: December 18, 2020, Available Online: December 19, 2020

\begin{abstract}
Due to handover failure, call drop occurs frequently. When a large number of incoming and handoff calls arrive at the same time, the performance of the conventional handoff algorithms may fall down. Moreover, multiple factors such as signal quality and available channels of cellular network can't be evaluated in conventional algorithms. When mobile station (MS) moves, the connection of MS with nearby base station (BS) has to be switched from one to adjacent station. In this case, unnecessary handoffs will be occurred due to lack of proper decision of handoffs or lack of consideration about signal quality with available free channels. As a result call drop will occur frequently. For performing handoff efficiently, fuzzy logic based handoff decision algorithm, adaptive handoff threshold level using neural network and priority based dynamic channel allocation algorithm using neuro-fuzzy system has been proposed in this work. These algorithms will mainly focus on the proper decision of handoff based on evaluating signal strength, available free channels, spectrum efficiency, MS speed and distance from BS so that unnecessary and inefficient handoffs can't be performed. Simulation revealed that using neuro-fuzzy system, the channel capacity, SIR and Handoff management were improved better than the others in terms of spectrum utilization efficiency, MS speed and SIR. The efficacy of the methodology has been proved by imitating the proposed model using MATLAB software.
\end{abstract}

Keywords: Fuzzy Logic; Neural Network; Neuro-Fuzzy; Dynamic Channel Allocation; SIR; Handoff.

This work is licensed under a Creative Commons Attribution-NonCommercial 4.0 International

\section{Introduction}

Call drop is the sudden and undesirable termination of successfully established calls [1]. For customer's satisfaction, call drop curtailment is very important issue in wireless cellular communication system. It is the right of the taxpayer customers to have flexible and Reliable communication tie-up, better telehealth and Tele-medicine opportunity, emergency virtual meeting and Security management system etc. Bindia and Aggarwal published a paper on various handoff strategies using fuzzy logic [1]. They integrated heterogeneous wireless environment to trigger the network selection process to perform handoff satisfactorily. Nyambati and Oduol published a paper on handoff decision in order to analyze the impact of fuzzy based algorithm in a cellular network [2]. The impact of utilizing the fuzzy logic system for handover decision making considering the global system for cell phone communication (GSM) network has analyzed. Atayero and Luka [3] proposed adaptive neurofuzzy inference system for dynamic load balancing using the soft computing in 3GPP LTE. In this paper, they didn't take any consideration of the network parameters such as signal to interference ratio, available channel capacity or signal strength.

From the previous surveys, handover has contemplated as the main reason for call dropping. Due to wrong decision and improper threshold level, unsuccessful handoff occurs frequently which will lead to the effect of call drop [4]. With compared to these literature studies, this paper has tried to deal with network parameters by which handoff problem can be minimized to reduce call drop.
So this paper will propound possible solution based on Artificial Intelligence system to find out the right decision point with a view to ensuring successful handoff and Channel allocation based on measuring the value of network parameters. Artificial neural network, fuzzy logic and Neuro-fuzzy systems are the major AI tools [5] that have applied in this work.

\section{Methodology}

Three methods have provided below to find out the proper decision point and handoff threshold level for occurring successful handoff.

\subsection{Two handoff level algorithm based on fuzzy logic}

On the basis of two handoff algorithm, the request of handoff is commenced, when the signal toughness drops under the first handoff altitude. At this altitude, when the upcoming signal is brawny, the handoff will take place. The call will be handed off, when the second handoff altitude is outstretched. The received signal strength (RSS) itself includes interference and carrier signal [3]. The algorithm of two handoff level based on fuzzy logic is shown in Fig. 1.

The current received signal strength varying from $-110 \mathrm{dbm}$ to $-48 \mathrm{dbm}$, signal to interference ratio varying from $12 \mathrm{db}$ to $24 \mathrm{db}$ and upcoming base station RSS are considered as the handoff decision matrices. These decision matrices are used as linguistic variables as shown in Table 1. Also handoff decision is considered as output of fuzzy inference system shown in Table 2. 
M. A. Islam, M. R. Hasan and A. Begum /JEA Vol. 01(04) 2020, pp 130-138

Table 1 Fuzzy variable for three inputs of two handoff level algorithm

\begin{tabular}{|c|c|c|c|c|}
\hline \multirow{2}{*}{$\begin{array}{c}\text { Serial } \\
\text { Number }\end{array}$} & \multirow{2}{*}{ Fuzzy Variable and Span } & \multicolumn{3}{|c|}{ Input Ranges } \\
\hline & & LOW & AVERAGE & HIGH \\
\hline 1. & $\begin{array}{c}\text { Current RSS } \\
-110 \mathrm{dBm} \text { to }-48 \mathrm{dBm}\end{array}$ & $-110 \mathrm{dBm}$ to $-88 \mathrm{dBm}$ & $-92 \mathrm{dBm}$ to $-68 \mathrm{dBm}$ & $-72 \mathrm{dBm}$ to $-48 \mathrm{dBm}$ \\
\hline \multirow{3}{*}{2.} & \multirow{3}{*}{$\begin{array}{c}\text { New BS RSS } \\
-110 \mathrm{dBm} \text { to }-48 \mathrm{dBm}\end{array}$} & $-110 \mathrm{dBm}$ to $-88 \mathrm{dBm}$ & $-92 \mathrm{dBm}$ to $-68 \mathrm{dBm}$ & $-72 \mathrm{dBm}$ to $-48 \mathrm{dBm}$ \\
\hline & & \multicolumn{3}{|c|}{ Input Ranges } \\
\hline & & POOR & MEDIUM & BETTER \\
\hline 3. & $\begin{array}{c}\text { SIR } \\
14 \mathrm{~dB} \text { to } 24 \mathrm{~dB}\end{array}$ & $14 \mathrm{~dB}$ to $19 \mathrm{~dB}$ & $17 \mathrm{~dB}$ to $21 \mathrm{~dB}$ & $20 \mathrm{~dB}$ to $24 \mathrm{~dB}$ \\
\hline
\end{tabular}

Table 2 Fuzzy variable for output of two handoff level algorithm

\begin{tabular}{|c|c|c|c|c|c|}
\hline $\begin{array}{c}\text { Serial } \\
\text { Number }\end{array}$ & Fuzzy Variable and Span & \multicolumn{4}{|c|}{ Output Ranges } \\
\hline 1. & Handoff Decision 0 to 1 & NO & WAIT & BE-READY & YES \\
\hline
\end{tabular}

Table 3 Rule base for different inputs amalgamation

\begin{tabular}{|c|c|c|c|c|}
\hline $\begin{array}{c}\text { Rule } \\
\text { Number }\end{array}$ & Current RSS & SIR & New BS RSS & HOD \\
\hline 1 & LOW & POOR & LOW & YES \\
\hline 2 & LOW & MEDIUM & LOW & BE-READY \\
\hline 3 & LOW & BETTER & LOW & BE-READY \\
\hline 4 & AVERAGE & POOR & LOW & YES \\
\hline 5 & AVERAGE & MEDIUM & LOW & BE-READY \\
\hline 6 & AVERAGE & BETTER & LOW & $\mathrm{NO}$ \\
\hline 7 & HIGH & POOR & LOW & YES \\
\hline 8 & HIGH & MEDIUM & LOW & BE-READY \\
\hline 9 & HIGH & BETTER & LOW & $\mathrm{NO}$ \\
\hline 10 & LOW & POOR & AVERAGE & YES \\
\hline 11 & LOW & MEDIUM & AVERAGE & BE-READY \\
\hline 12 & LOW & BETTER & AVERAGE & BE-READY \\
\hline 13 & AVERAGE & POOR & AVERAGE & YES \\
\hline 14 & AVERAGE & MEDIUM & AVERAGE & BE-READY \\
\hline 15 & AVERAGE & BETTER & AVERAGE & AVERAGE \\
\hline 16 & HIGH & POOR & AVERAGE & YES \\
\hline 17 & HIGH & MEDIUM & AVERAGE & WAIT \\
\hline 18 & HIGH & BETTER & AVERAGE & $\mathrm{NO}$ \\
\hline 19 & LOW & POOR & HIGH & YES \\
\hline 20 & LOW & MEDIUM & HIGH & BE-READY \\
\hline 21 & LOW & BETTER & HIGH & WAIT \\
\hline 22 & AVERAGE & POOR & HIGH & YES \\
\hline 23 & AVERAGE & MEDIUM & HIGH & BE-READY \\
\hline 24 & AVERAGE & BETTER & HIGH & $\mathrm{NO}$ \\
\hline 25 & HIGH & POOR & HIGH & YES \\
\hline 26 & HIGH & MEDIUM & HIGH & WAIT \\
\hline 27 & HIGH & BETTER & HIGH & $\mathrm{NO}$ \\
\hline
\end{tabular}


Sugeno fuzzy inference system [5] will be used to process these matrices parameters. The output of the FIS means the membership value of handoff decision shown in Table 2. Based on the different amalgamation of the three inputs and one output parameters, the different twenty seven rules can be conceived as shown in Table 3.

The handoff decision parameter [6] depending on the three input parameters such as RSS of current base station, signal to interference ratio and RSS of new base station can be assessed as shown in Fig. 2.

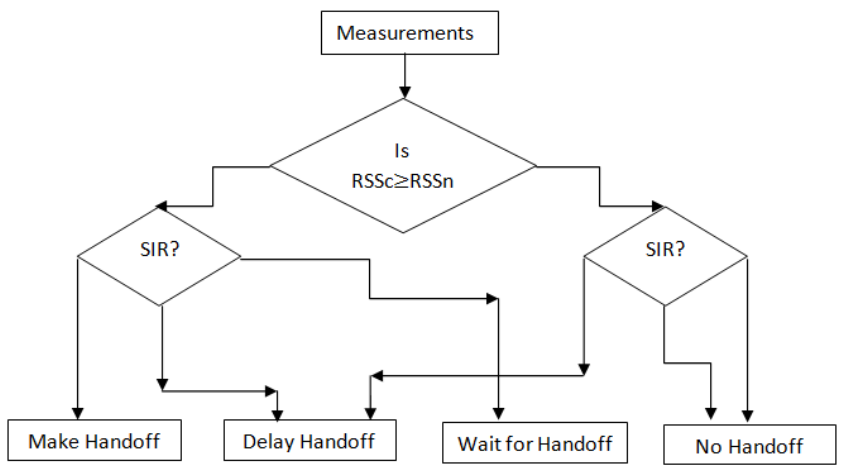

Fig. 1 Two handoff level algorithm based on fuzzy logic.

2.2 Artificial Neural network based Adaptive handoff threshold level algorithm

To manifold problems, artificial neural networks can be applied. The utility of three parameters [7] such as slope ratio, signal to interference ratio and the number of available free channels can be calculated using fuzzy logic system. The threshold value for handoff will be adopted to fortify successful handoff according to the value of such parameters.
Fig. 3 shows the training data sets considered as input data sets for learning. These inputs are applied to the fuzzy logic system and the FLS outputs can be computed. The ranges of slope ratio, signal to interference ratio are from 0 to $1,14 \mathrm{db}$ to $21 \mathrm{db}$ and 0 to 12 . The test data to validate the model is shown in Fig. 4. The FLS output data will serve as the target output shown in Fig. 5.

\subsection{Neuro-fuzzy based Dynamic Channel Allocation Scheme}

In this work, DCA [8] can be used to flourish a channel allocation policy. Neuro-adaptive learning mechanisms render a procedure for employing dynamic channel allocation stratagem to grasp information about a data set. For a given input or output data set, the toolbox function ANFIS contrives a fuzzy inference system (FIS). Back propagation algorithm can be used to tune the parameters of membership function [4]. By this process, the permission to learn from the modeling data will be obtained. Through the learning action, the related parameters can be changed.

In fuzzy control toolbox, a handy command called ANFIS exists. An optimization stratagem will be given by this command to find the parameters in the fuzzy system that will be the best to fit the data. In the first juncture, the crisp variables, spectrum utilization efficiency, speed of secondary user, distance from base station and in the fuzzification action, RSS will be transmuted into linguistic variables. The four input variables can be mapped into fuzzy altitude of fuzzy sets through fuzzification. A sigmoidal membership function has used as an activation function in this work. Center of gravity [5] method has used in defuzzification method. A typical neuro-fuzzy system is shown in Fig. 6. The rule evaluation for dynamic channel allocation scheme is shown in Fig. 7. Considering different combinations of four matrices, different outputs are generated to be trained in neuro-fuzzy system.

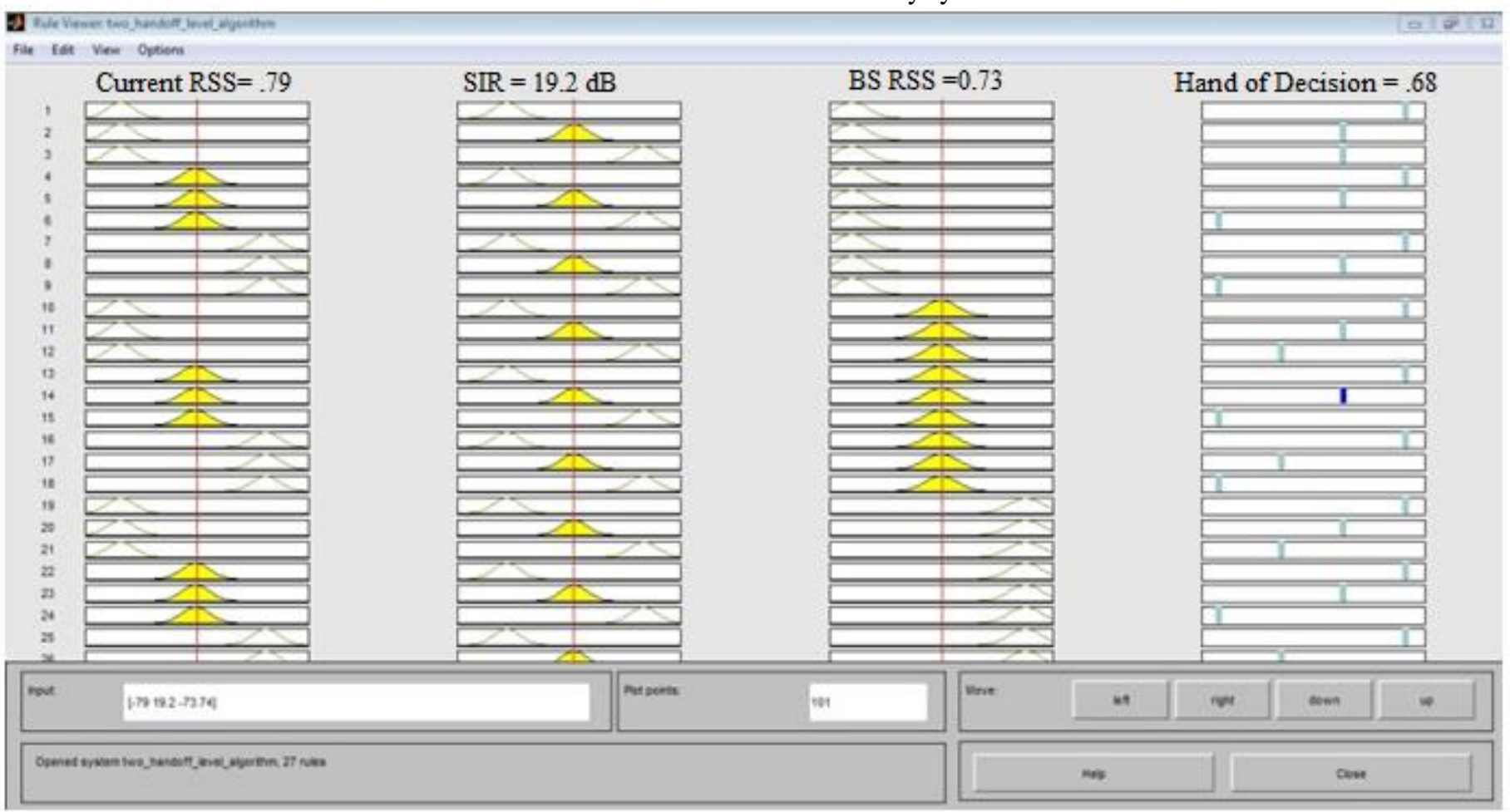

Fig. 2 Rule evaluation for determining handoff decision level 


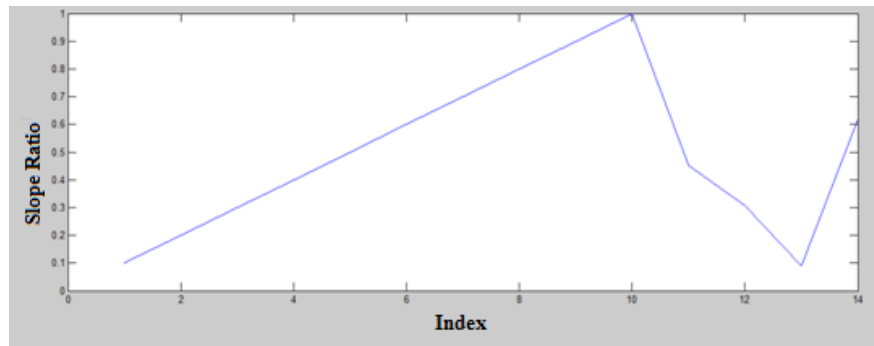

(a)

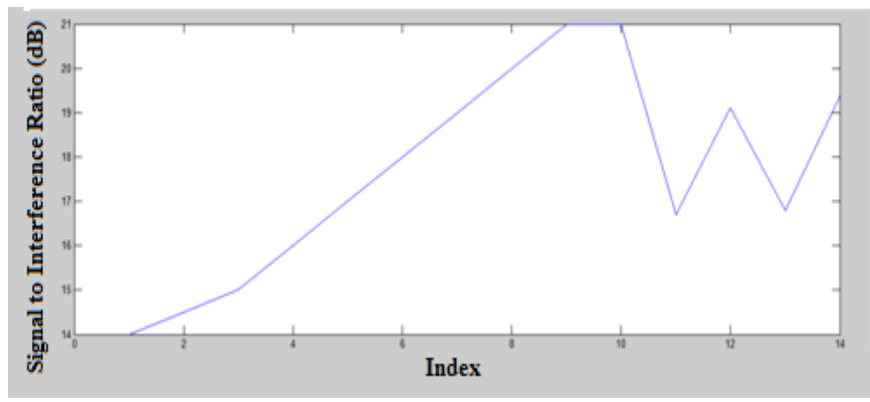

(b)

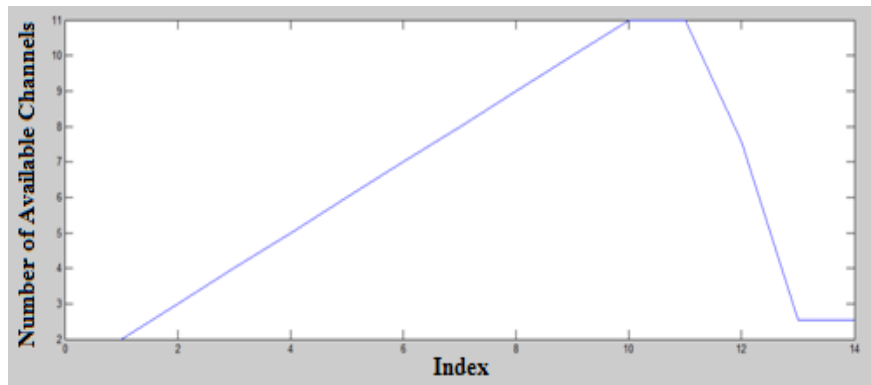

(c)

Fig. 3 Training data for Neural Networks

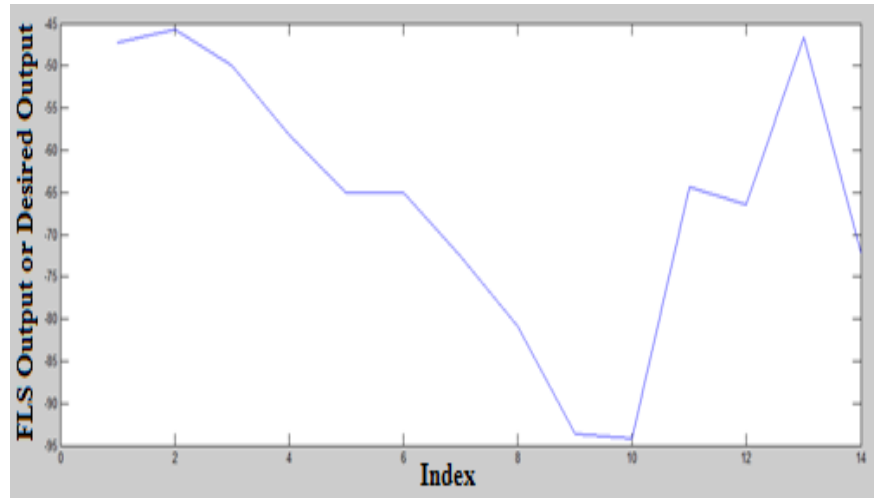

Fig. 5 FLS output or Desired Output

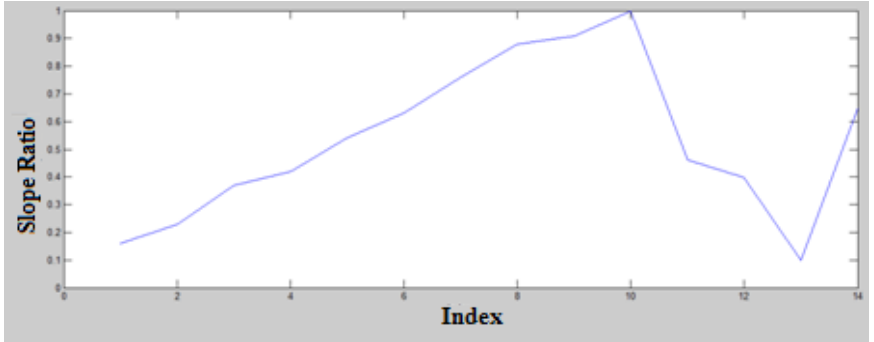

(a)

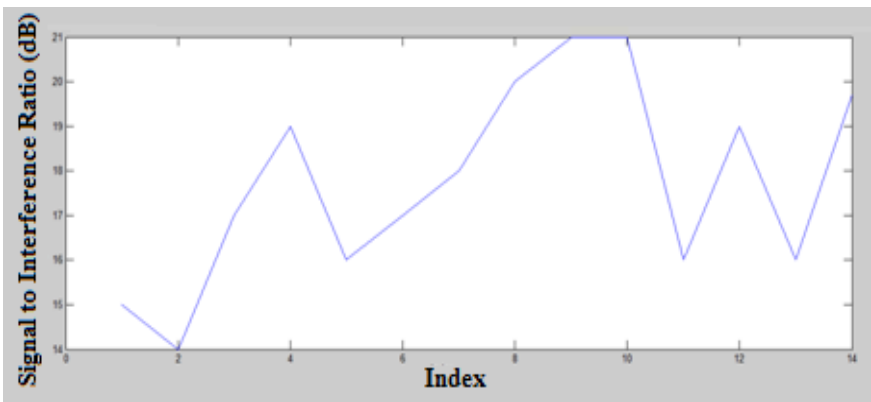

(b)

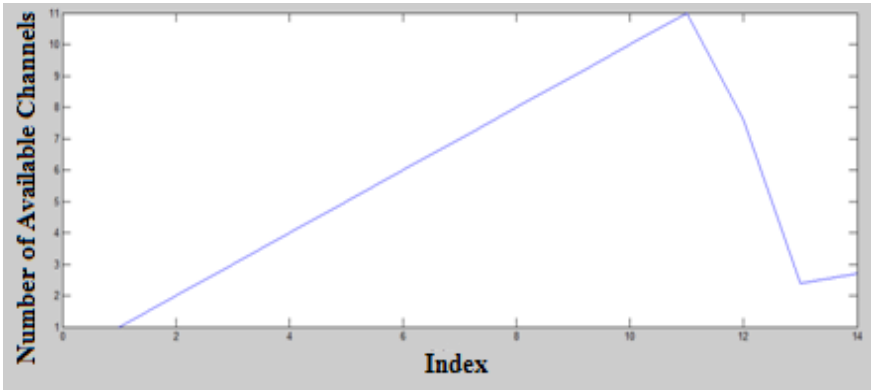

(c)

Fig. 4 Test data for neural network

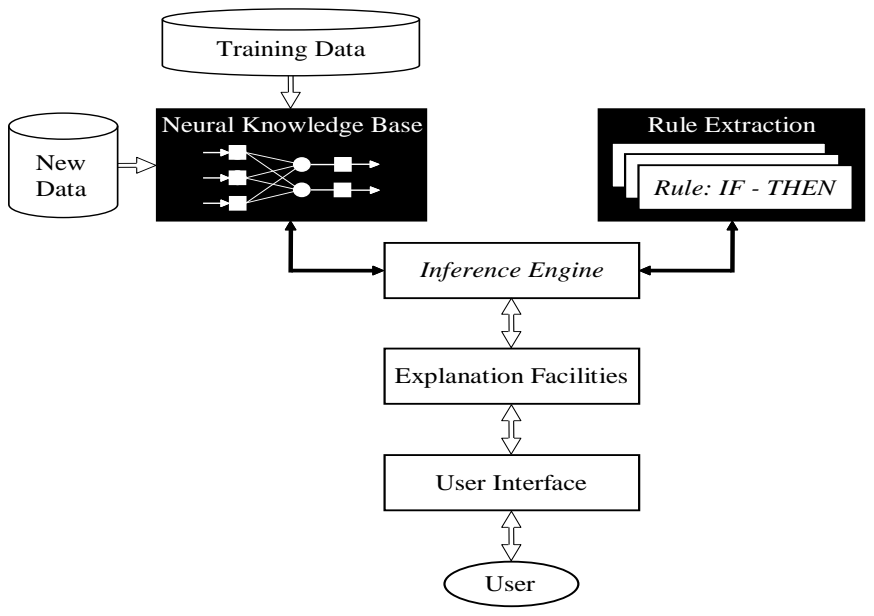

Fig. 6 Neuro-fuzzy system 


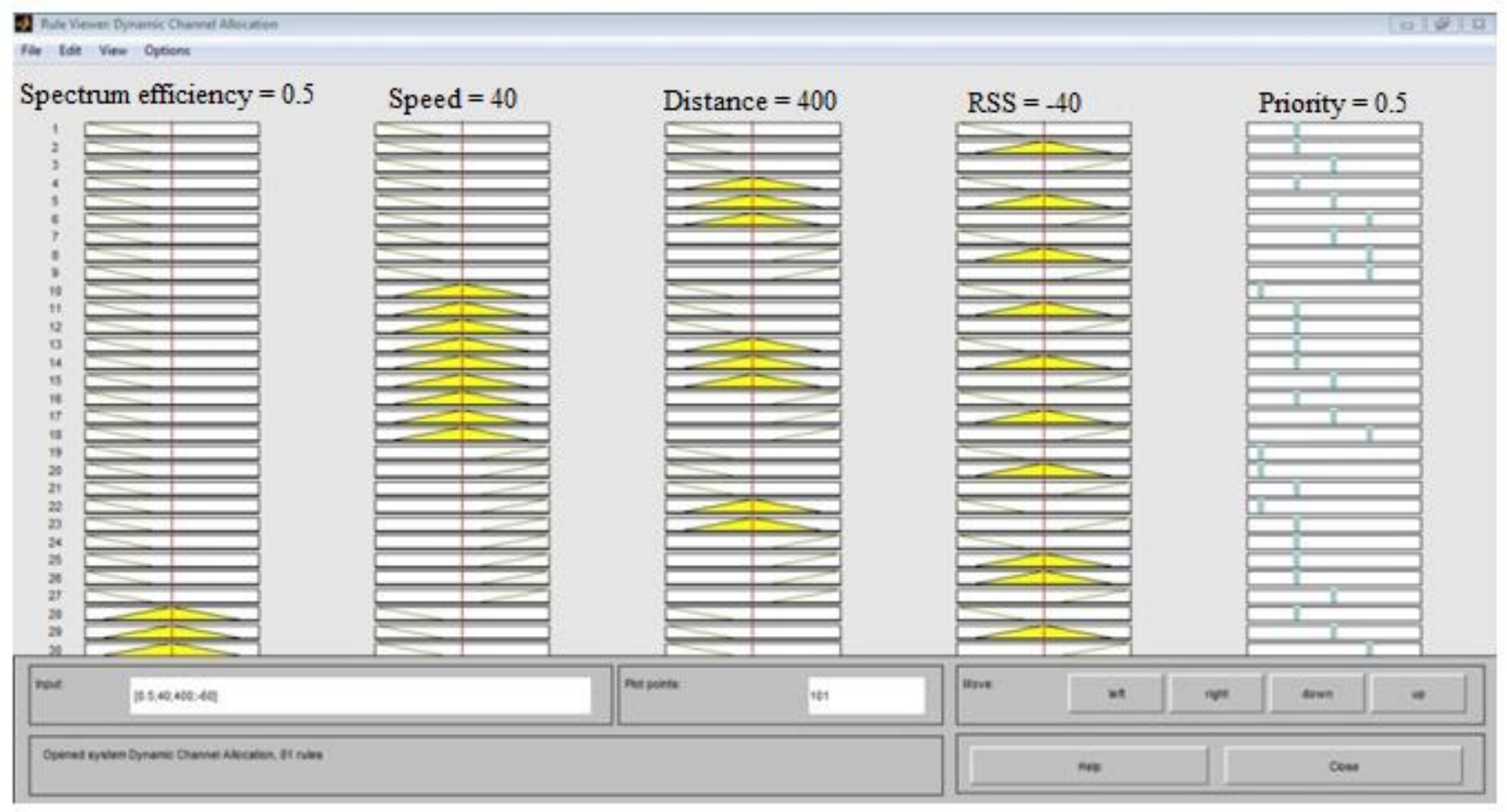

Fig. 7 Rule evaluation for dynamic channel allocation

\section{Results}

3.1 Two handoff level algorithm based on fuzzy logic

The following alliance can be procured from the two handoff level algorithm based on fuzzy logic. As the current received signal strength decreases after a certain period, the handoff decision probability increases drastically shown in Fig. 8.

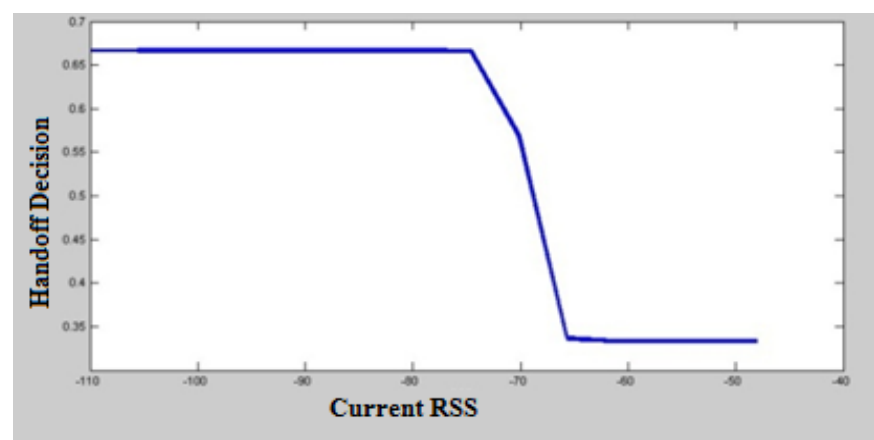

Fig. 8 Relationship between Current received signal strength and Handoff decision.

As the RSS of upcoming base station increases, the probability of switching from old BS to new BS increases shown in Fig. 9. Normally SIR drops as a function of distance. Besides due to interference, SIR can be decreased which is shown in Fig. 10. As the SIR decreases, the probability of handoff increases which switches the MSC from current BS to new BS.

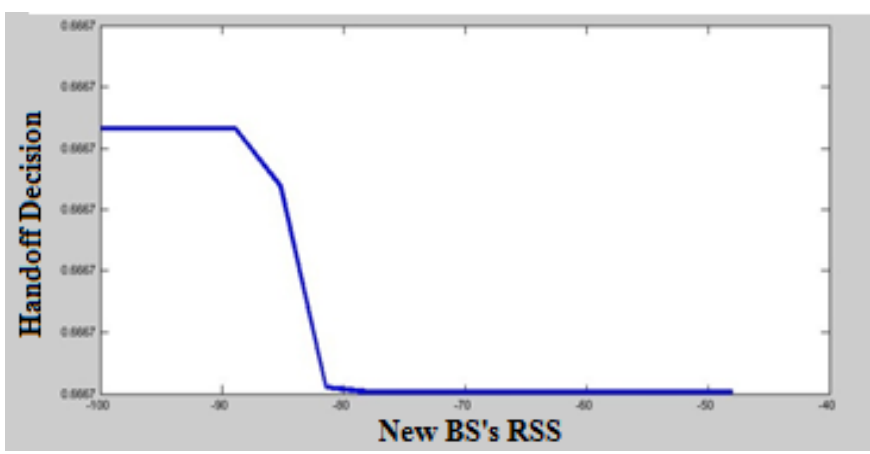

Fig. 9 Relationship between new BS received signal strength and Handoff decision.

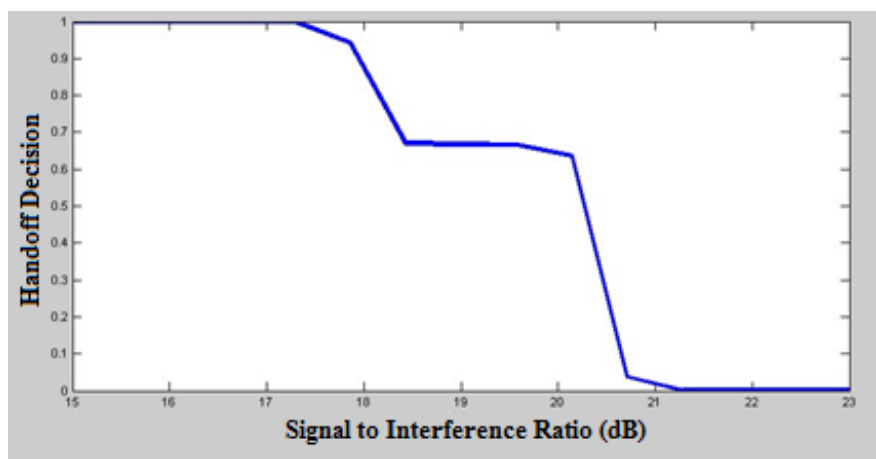

Fig. 10 Relationship between signal to interference ratio and Handoff decision. 


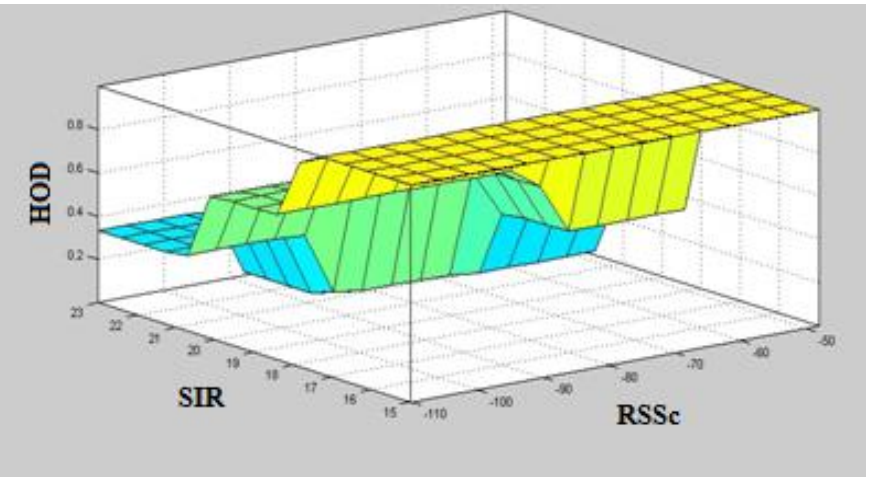

Fig. 11 Surface plot for current received signal strength and signal to interference ratio versus Handoff decision.

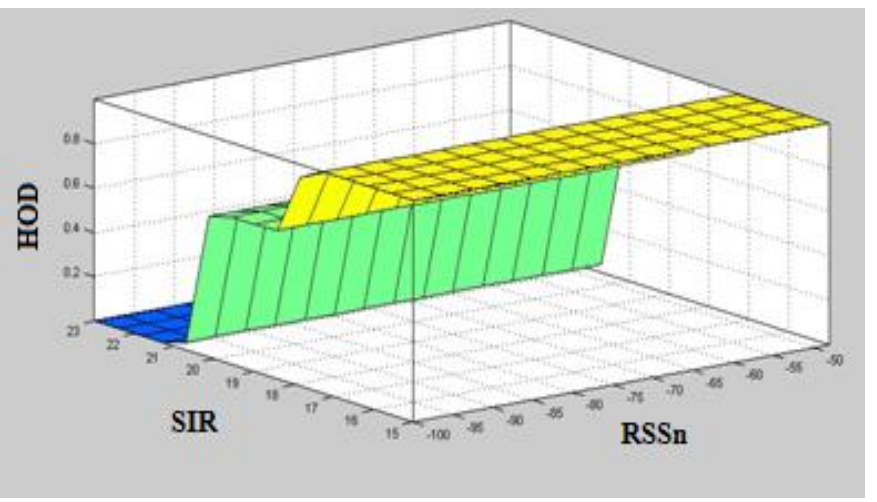

Fig. 12 Surface plot for new base station received signal strength and signal to interference ratio versus Handoff decision.

Fig. 11 represent the surface plot of handoff with respect to current RSS and SIR. As current RSS and SIR are decreasing, handoff is increasing. Fig. 12 represents the surface plot of handoff with respect to new RSS and SIR. As new RSS increases and SIR decreases, handoff increases gradually. Fig. 13 represents the surface plot of handoff with respect to new RSS and current RSS. The current RSS drops as a function of distance and after a distance the new BS RSS increases. When the new BS RSS is found greater than the old RSS, then the handoff process will be executed. When the fuzzy based two handoff level algorithm is used, the handoff decision can be properly taken and thus the channel performance and capacity is increased.

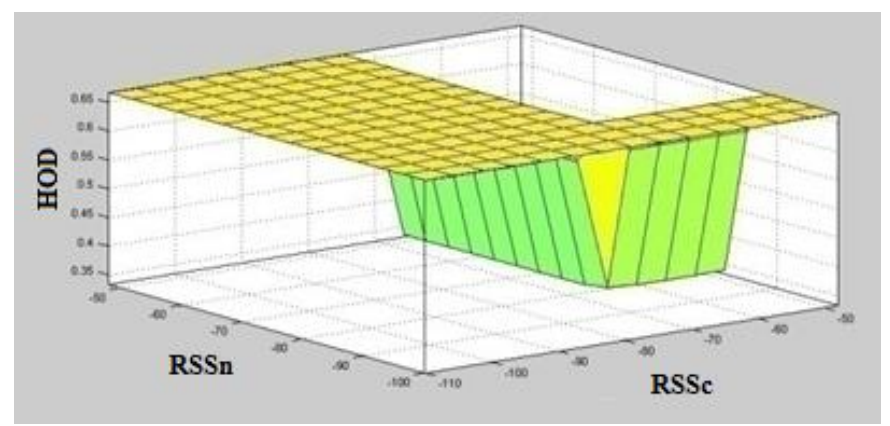

Fig. 13 Surface plot for current received signal strength and new base station received signal strength versus Handoff decision.
3.2 Artificial neural network based adaptive handoff threshold level algorithm

For different amalgamation of inputs data, the FLS output data is the desired output. Then the neural network is trained with these inputs and output. When the network is satisfactorily trained, any amalgamation of the test data will follow the desired output. The performance evaluation of actual and multi-layer protocol output is shown in Fig. 14. Blue line indicates the target or desired output, where the green line is the predicted output with 10 neurons, cyan line is the predicted output with 12 neurons, and red line is the predicted output with 15 neurons. As the number of neurons increase, the chance of getting authoritative desired output also increases with the cost of complexity. The red line is more close to the target output than the others.

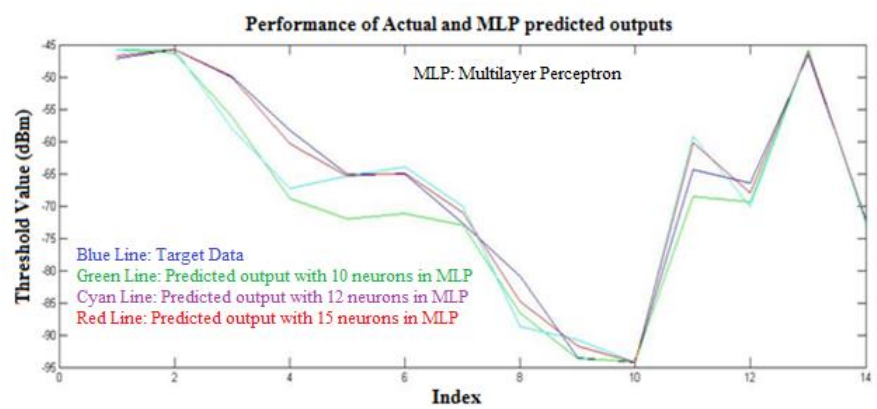

Fig. 14 Performance Evaluation of actual and MLP output.

Thus the fuzzy logic output is trained into the neural network so that the threshold level of handoff occurrence can adapt according to miscellaneous situations and gives the output close to the desired output. Thus the handoff process can be successfully accomplished. Hence the channel capacity is increased and call drop probability due to handoff is reduced. The relationship between different inputs can be discussed.

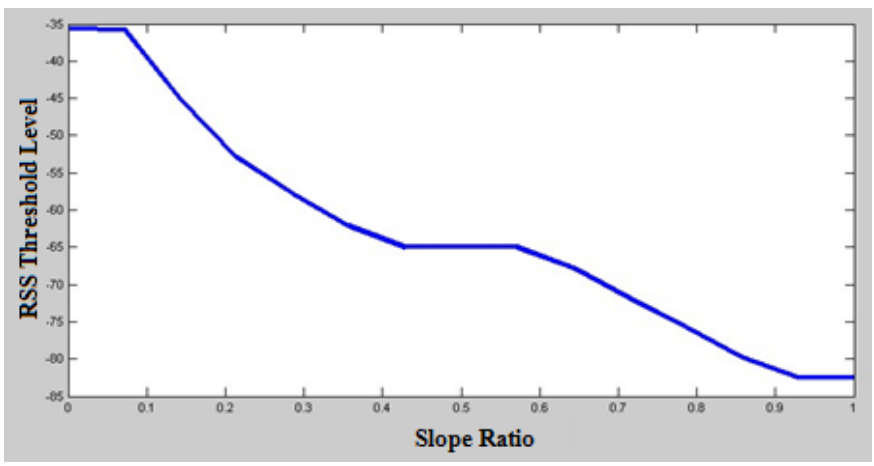

Fig. 15 Slope ratio Vs RSS threshold level

For different values of $\theta_{1}$ and $\theta_{2}$, the various values of slope ratio are obtained. When $\theta_{2}$ is greater than $\theta_{1}$, then handoff takes place. As $\theta_{2}$ is decreased, the received signal strength is increased and also the slope ratio is increased which is shown in Fig. 15. As the slope ratio increases, the chance of handoff decreases which is shown in figure. So the threshold value should be set up in such a way that the no handoff can be performed unnecessarily. 


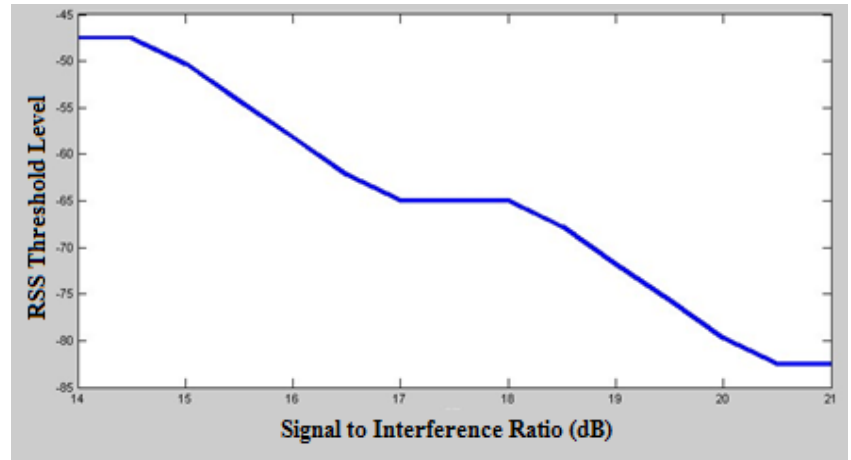

Fig. 16 SIR Vs RSS threshold level

As the signal to interference ratio is decreased, then the threshold level for handoff occurrence should be set to high value so that no call drop can be occurred. The tie-up between them is shown in Fig. 16.

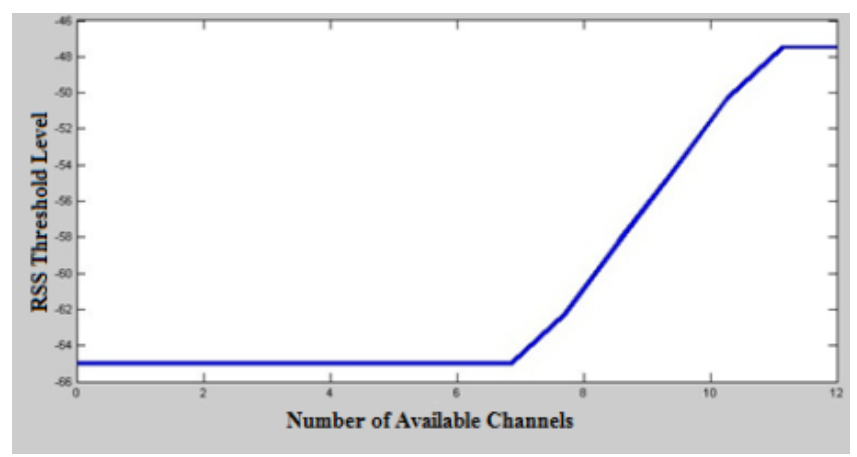

Fig. 17 Relationship between available free channels and RSS threshold level for handoff decision.

As the available free channels are decreased, then the threshold level for handoff occurrence must be set to possible lower value, so that the call can continue for the last moment without handoff as can as possible shown in Fig. 17.

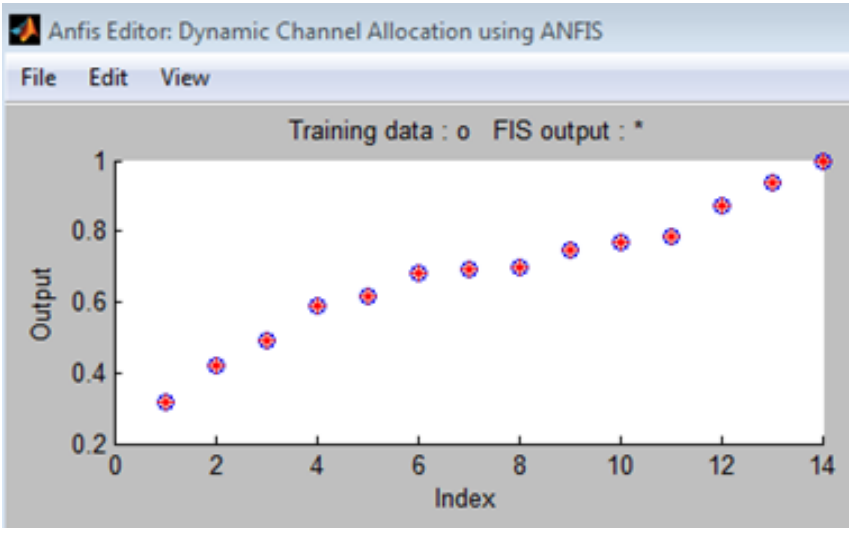

Fig. 18 Testing FIS against training data

\subsection{Neuro-fuzzy based dynamic channel allocation scheme}

To assemble the output of artificial neuro-fuzzy inference system that game the desired or wanted data, all the adaptable parameters have tuned. For this purpose, the learning action has been utilized. An amalgamation of learning algorithm has been adopted in such a way that the parameters of input-output membership functions can be adjusted to ameliorate the training efficiency.
The mean square error procedure has utilized to optimize the consequent parameters with keeping the antecedent parameters fixed. Then the back propagation algorithm has used to tune the surmise parameters, when the consequent parameters are updated.

When the training error result is acceptable, then the performance test of fuzzy inference system against either training, testing or checking data can be evaluated which is shown in Fig. 18 and

Fig. 19. Click test now and the following display will be seen.

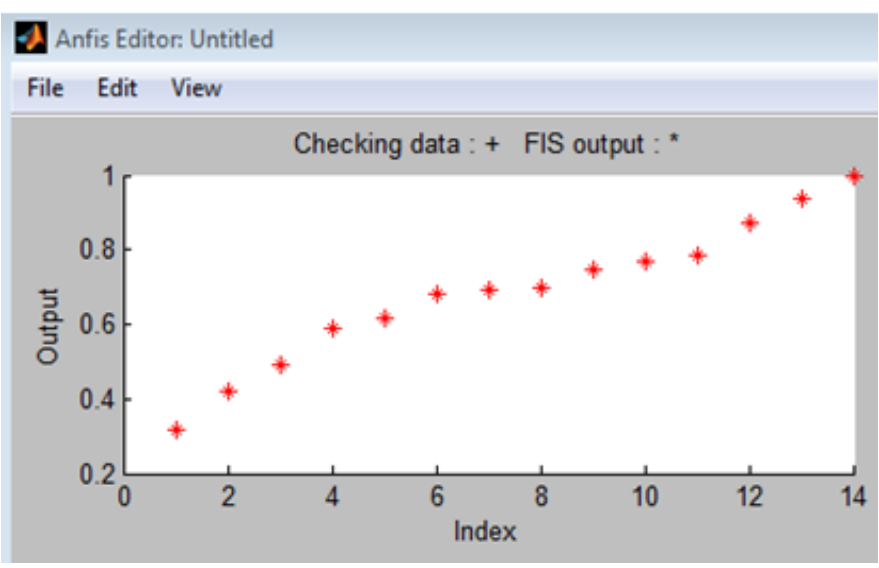

Fig. 19 Checking data

The training aids to select the quintessential rule to be fired. The relationship between the tuned parameters can be entrenched as follows:

Fig. 20 shows that as the spectrum efficiency increases, the allocation priority increases. Thus the channel can be allocated according to the user holding higher priority factor. Fig. 21 shows that when the secondary user speed is decreased, then the priority factor is increased. As the speed of secondary user increases, the ability to detect the primary user decreases. So allocation priority will be high with slow speed.

Fig. 22 shows that the closest between secondary user and primary user, the possibility of interference to primary user is greater. So allocation priority is high with far distance from base station.

Fig. 23 shows that with high received signal strength, the allocation priority is high. Thus the higher RSS value of secondary user can be allocated to new channel with high preference. Fig. 24 shows that the priority factor is higher with the higher value of spectrum efficiency and the distance. Depending on the higher priority factor, the channel can be allocated to the specific user holding high priority factor.

Fig. 25 shows that the priority factor is higher with the higher value of spectrum efficiency and the received signal strength. Depending on the higher priority factor, the channel can be assigned to the specific user. Fig. 26 shows that with smaller spectrum efficiency and faster speed, the priority factor is decreased. Unlike higher priority factor, the channel is not assigned to the specific secondary user normally.

Fig. 27 shows that with higher spectrum efficiency and slower speed, the priority factor is increased. With higher priority factor, the channel is assigned to the specific secondary user. The available channel allocation table is shown in Table 4. 


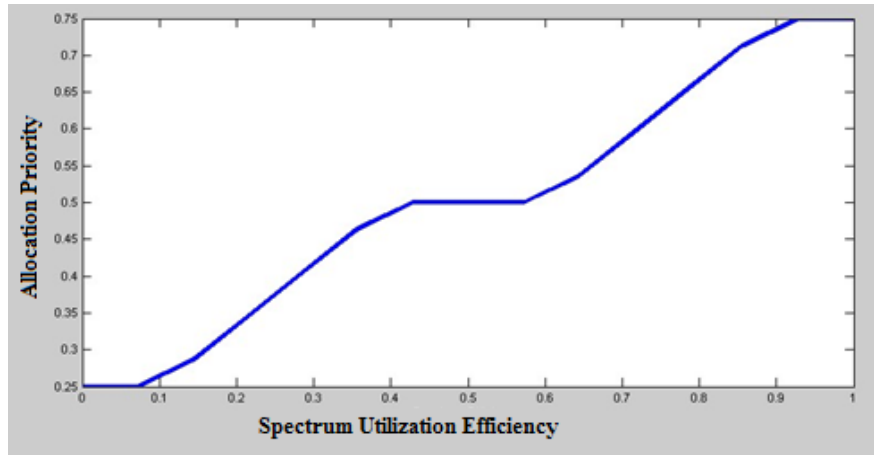

Fig. 20 Relationship between spectrum utilization efficiency and allocation priority

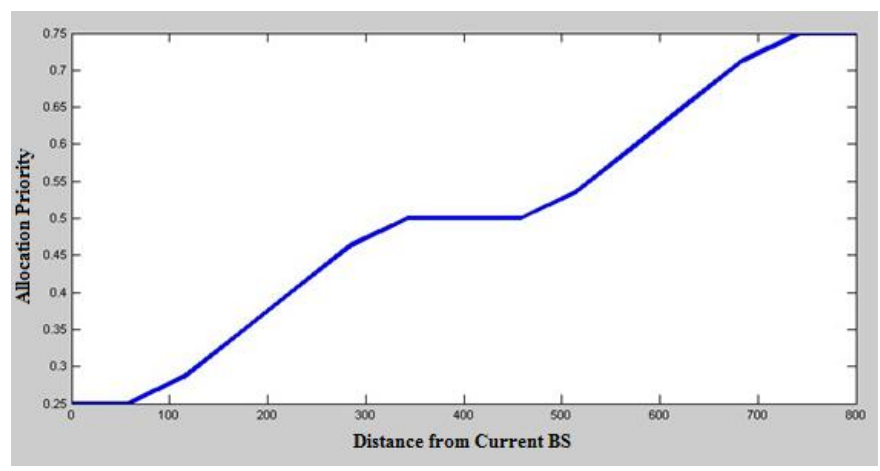

Fig. 22 Relationship between distance from BS and allocation priority

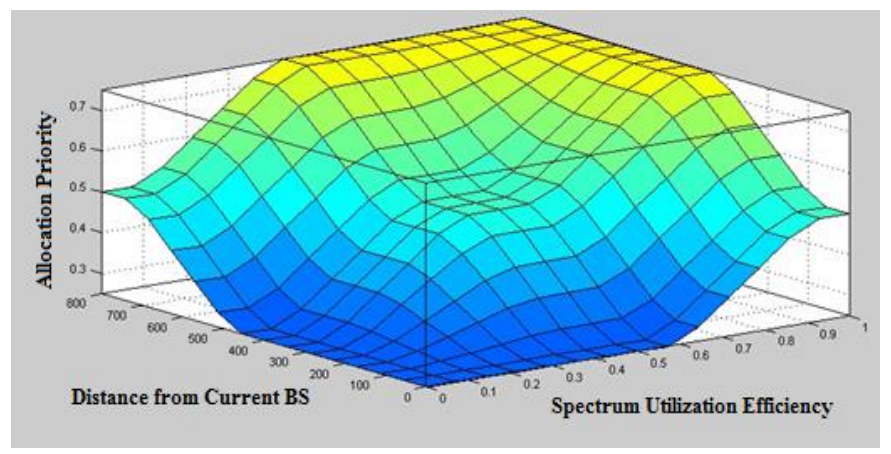

Fig. 24 Relationship between distance, spectrum efficiency and allocation priority

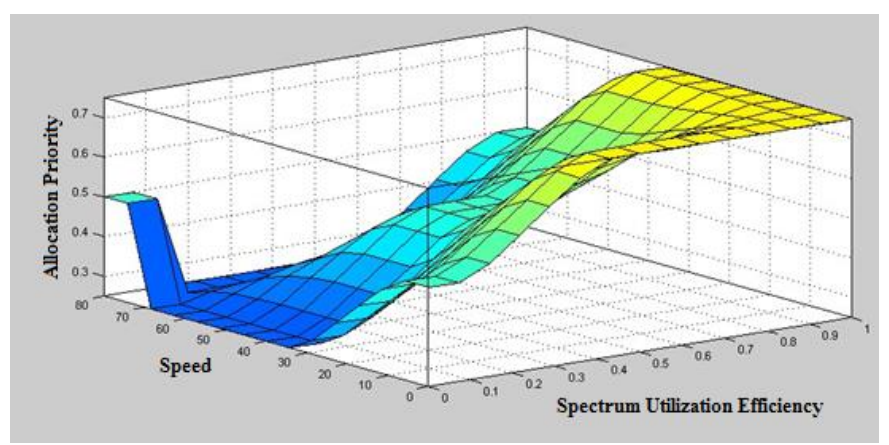

Fig. 26 Relationship between speed, spectrum efficiency and allocation priority

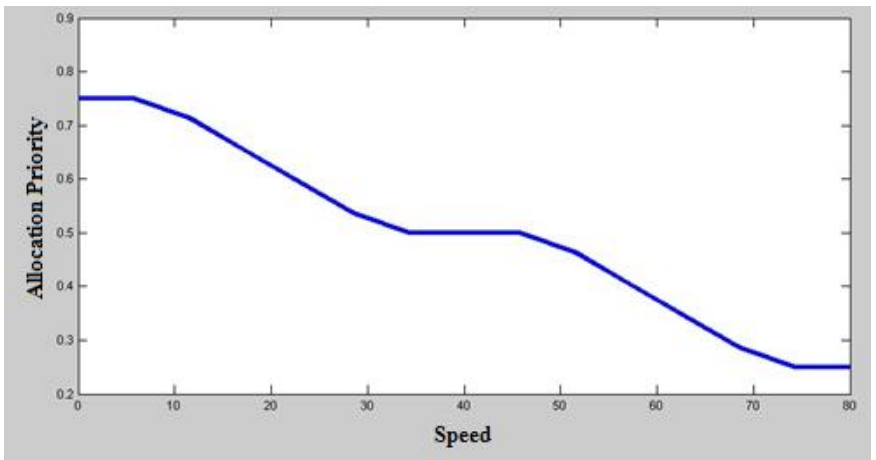

Fig. 21 Relationship between secondary user speed and allocation priority

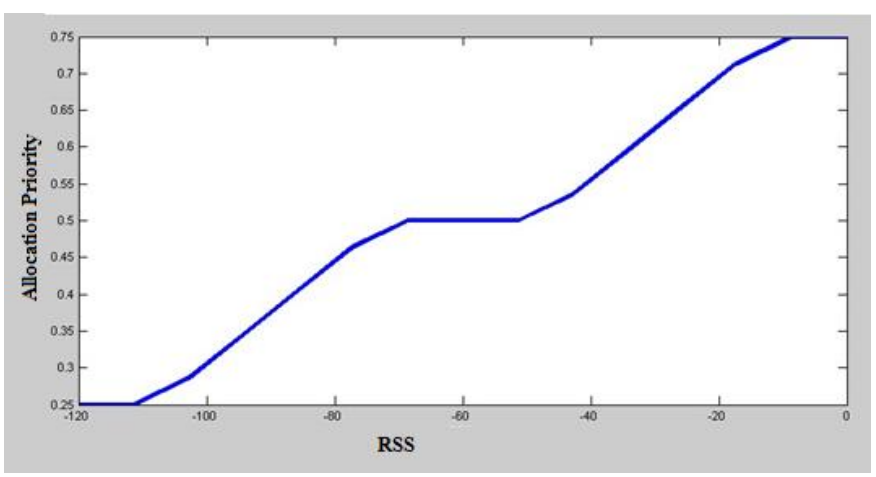

Fig. 23 Relationship between received signal strength and allocation priority

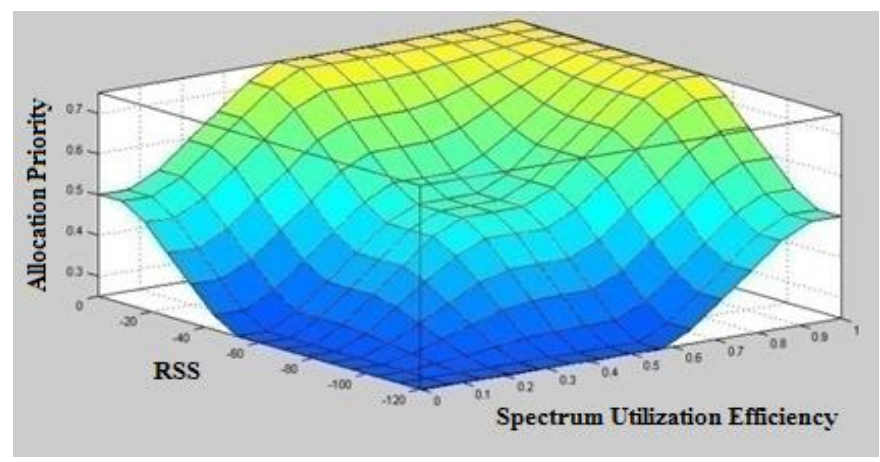

Fig. 25 Relationship between RSS, spectrum efficiency and allocation priority

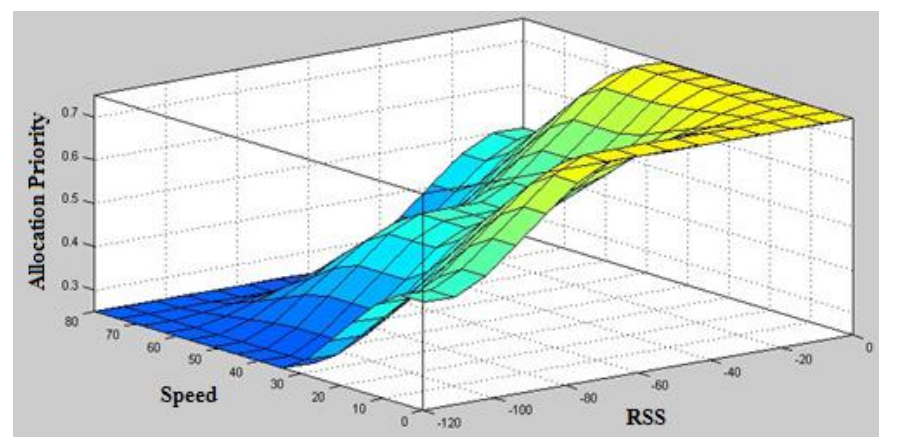

Fig. 27 Relationship between speed, RSS and allocation priority 
Table 4 Channel allocation table

\begin{tabular}{|c|c|c|c|c|}
\hline & Channel-1 & Channel-2 & Channel-3 & Channel-4 \\
\hline SU 1 & .382 & 0 & 0 & $\ldots$. \\
\hline SU 2 & .769 & 0 & .241 & $\ldots$. \\
\hline SU 3 & .8558 & 0 & .492 & $\ldots$. \\
\hline SU 4 & .718 & .608 & 0 & $\ldots \ldots$ \\
\hline$\ldots$. & $\ldots$ & $\ldots$ & $\ldots \ldots$ & $\ldots$. \\
\hline
\end{tabular}

After calculation by fuzzy inference system, a priority factor of secondary user (SU) has generated and the channel will be allocated to the SU holding the highest priority factor. From, to use the channel, SUs contend with the priority factor generated by FIS. With the help of information existing in the channel allocation table, SU with higher priority factor will get the right to use the channel. There are four SUs shown in Table 4 and they compete for allocating channel 1 , but due to high priority factor, channel 1 will be allocated to SU4 having the highest priority factor of 0.8558 . The others may be concluded by the same procedure such as channel 2 will be allocated to SU2, channel 3 allocated to SU3.

\section{Conclusion}

In this research, handoff algorithm based on fuzzy logic and neural network have introduced that utilize the advantages of multi-criteria handoffs. With the help of well-known delicacy of handoff parameters, these handoff techniques have implemented to find out the proper decision. In two handoff level algorithm based on fuzzy logic technique, three network parameters have taken as linguistic variables of three degree of memberships such as LOW, AVERAGE and HIGH to generate output for handoff decision. Decision phase has classified into four levels such as NO, WAIT, BE-READY and YES based on the fuzzy rules inserted in the sugeno inference mechanism. In Artificial neural network based on adaptive handoff threshold level algorithm, three parameters such as slope ratio, available free channels and signal to interference ratio have taken into consideration to find the suitable point of handoffs decision. Different data sets of these parameters have used as linguistic variables and fuzzy rules have inserted to produce target outputs. Using this fuzzy model, different data sets have generated to be used as training data in neural network. Then any arbitrary level of these parameters will follow the target outputs to identify the decision point of handoffs. In dynamic channel allocation scheme using neurofuzzy model, four network parameters have considered such as distance from BS, MS speed, spectrum efficiency and received signal strength to determine priority factor for allocation of channels of the switching requested status of the mobile cellular network. This approach ameliorates channel capacity and signal quality in terms of quality of service and hence reduces call drop. The peril of call drops is very scathing and needs to be reconciled very soon. Other methods like Vertical handoff algorithm, BER reduction, RF optimization and Multiple Input Multiple Output antenna also evince to be worthwhile in achieving this target. In the future, the plan can be taken to flourish a systematic manner for priority based handoff mechanism using neural network.

\section{References}

[1] Bindia. Aggarwal, B. 2017. Various Handoff Strategies using Fuzzy Logic. International Journal of Scientific Research and Management, 5(03), pp 5199-5203.

[2] Nyambati, E.T. and Oduol, V.K., 2017. Analysis of The Impact of Fuzzy Logic Algorithm On Handover Decision in A Cellular Network. International Journal for Innovation Education and Research, 5(5), pp.46-62.

[3] Atayero, A.A. and Luka, M.K., 2012. Adaptive neurofuzzy inference system for dynamic load balancing in 3GPP LTE. International Journal of Advanced Research in Artificial Intelligence, 1(1), pp.11-16.

[4] Zeng, Q.A. and Agrawal, D.P., 2002. Handoff in wireless mobile networks. Handbook of wireless networks and mobile computing, 28, pp.1-25.

[5] Mir, G.M. and Shah, N.A., 2009. Decentralized Handoff for Microcellular Mobile Communication System using Fuzzy Logic. In World Acadamy of Science, Engineering and Technology, pp. 866-870.

[6] Garg, V., 2010. Wireless communications \& networking. Elsevier.

[7] Wang, Y.H. and Liao, S.L., 2017, March. Applying a fuzzy-based dynamic channel allocation mechanism to cognitive radio networks. In $201731^{\text {st }}$ International Conference on Advanced Information Networking and Applications Workshops (WAINA) (pp. 564-569). IEEE.

[8] P.Ramu. 2014. Optimized Handoff Scheme using Fuzzy Logic.Report, Jaya Institute of Technology, Chennai, India. 